\title{
The Ecological Formulation of a Pesticide Manufactured by JSC Schelkovo Agrohim (Russia) on the Example of the Herbicide Zontran CSC.
}

\author{
Salis Karakotov, Elena Zheltova and Sergey Klishin \\ JSC Schelkovo Agrohim, Moscow Region, Schelkovo 141101, Russian Federation
}

\begin{abstract}
The results of studying the effectiveness of the herbicide Zontran CSC, containing the active substance metribuzin $250 \mathrm{~g} / \mathrm{L}$ in a preparative form (formulation) named colloidal solution concentrate (CSC) are presented. Experiments were carried out for two years in 2011-2012 on soybean crops with mixed type of weeds: annual dicotyledonous and annual grasses. The biological effectiveness of Zontran CSC was compared with a product containing metribuzin $700 \mathrm{~g} / \mathrm{kg}$ in a formulation wettable powder (WP). It was established that the pre-emergence application of Zontran CSC on soybean crops at a dose rate of $1.2 \mathrm{~L} / \mathrm{ha}$ was comparable to the biological effect of the product, consisting of metribuzin at 2.3 times more, but in the WP formulation. It was observed that Zontran CSC in comparison with the product containing a higher amount of metribuzin, but in the WP formulation, was at the level, and in some cases exceeded the biological effectiveness of this product. Thus, the formulation of the CSC allowed, without loss of biological effectiveness and with obtaining reliable yield increases, reducing the amount of metribuzin per unit of cultivated area, which makes the use of pesticide environmentally friendly, since it minimizes its release into the environment.
\end{abstract}

Key words: Herbicides, metribuzin, CSC, formulation, soybean.

\section{Introduction}

Intensive application of the mineral fertilizers and pesticides is the cause to the yearly entry into the biosphere of various chemicals. In this regard, the problem of environmental protection, especially when using pesticides, is important [1]. The use of pesticides ensures to save crop yield by controlling the number of harmful objects. However, applying pesticides during or before the vegetation periods leads to the fact that chemicals that can adversely affect the soil biota and terrestrial organisms are released into the environment and accumulated in the form of residues in agricultural products. Therefore, reducing the pesticide accumulation on nature is actual and significant.

Biological efficacy of the products depends on the active substances in their composition, as well as the

Corresponding author: Sergey Klishin, $\mathrm{PhD}$, research field: biology. amount of active ingredient that interacts with the harmful objects, which is determined by the dose rate of the pesticide. Preparative form of pesticide (formulation) is the composition of the active substance of the pesticide and auxiliary substances, ensuring the delivery of the active substance to the target object.

The different biological efficacy is achieved with the same active ingredient and its equal amount but depending on the type of formulation. The dose rate decreasing of active ingredients may lead to a decrease in biological effectiveness.

JSC Schelkovo Agrohim set a goal to create products that would have a biological efficiency comparable to the level of traditionally used pesticides, but contained a smaller amount of the same active ingredient per unit of cultivated area in order to minimize the pesticide environmental accumulation. This effect was supposed to be obtained by means of a new formulation, which is a special case of the 
emulsion concentrate (EC) - the colloidal solution concentrate (CSC). CSC is an EC which, when preparing the working solution, forms a colloidal system. A colloid system is a system in which discrete particles, droplets or bubbles of a dispersed phase, having a size in at least one dimension from 1 to 100 $\mathrm{nm}$, are distributed in another phase, usually continuous, different from the first in composition or aggregative state and called dispersion phase. The popularity of colloidal systems lies in their unique properties that are transmitted to colloid-based products: the system is homogeneous, there is no sediment (the product has a transparent colour), a high coverage ratio, wetting is ensured, which cause to a significant increase in the degree of absorption of active substances, and as a result, optimization of biological activity.

A whole line of the products of CSC formulation was created, and one of them is the herbicide Zontran CSC containing $250 \mathrm{~g} / \mathrm{L}$ of metribuzin.

Metribuzin is included as an active ingredient in many pesticides. It is used pre- and post-emergence to control weeds on following crops: potatoes, wheat, tomatoes, carrots, soybeans, and sugarcane. The mechanism of action is based on the inhibition of the photosystem II [2].

The objective of this research was to study the biological effectiveness of the herbicide Zontran CSC and compare it with its analogue (hereinafter referred to as Analog) containing $700 \mathrm{~g} / \mathrm{kg}$ of metribuzin in the formulation wettable powder (WP).

\section{Material and Methods}

Biological trials were carried out for two years in 2011-2012 in three regions of the Russian Federation on the soybean varieties Annushka, Bara, Svetlaya. The Zontran CSC was applied pre-emergence, on vegetative weeds with dose rates of $0.6,0.8$ and 1.2 L/ha, which corresponds respectively to the application of metribuzin 150, 200 and $300 \mathrm{~g} / \mathrm{ha}$ consequently. At the application time, weeds were in the phase of cotyledons - two leaves.

The biological efficiency of Zontran CSC was compared with the Analog containing $700 \mathrm{~g} / \mathrm{kg}$ metribuzin, formulation WP, in dose rates of 0.5 and $1.0 \mathrm{~kg} / \mathrm{ha}$, which corresponds to 350 and $700 \mathrm{~g}$ of metribuzin on ha respectively. The trials were provided in accordance with the Guidelines for Registration Testing of Herbicides in Agriculture [3] under conditions of small plots of $0.25 \mathrm{~m}^{2}$ in the mixed type of weediness. The names of weed plant species were given in accordance with the work of Mayevsky Flora of the Middle Zone of the European Part of Russia [4].

The study was conducted by an independent source namely All-Russian Institute for Plant Protection (VIZR), and the results of this research Zontran CSC received an extension of official registration of pesticides in the Russian Federation for soybeans.

The determination of the particle size of the Zontran CSC was carried out on a Photocor Compact instrument using photon correlation spectroscopy. Particle size analysis of the Analog (WP) has been done on a Fritsch Analysette 22 MicroTec Plus instrument by laser diffraction. The surface tension of each of the products was determined by the method of ring detachment on a Kruss K6 tensiometer.

\section{Results and Discussion}

In case of pre-emergence application of the Zontran CSC in soybean crops following weeds were effectively suppressed: Stellaria media L. (85\%), Amaranthus retroflexus L. (94.6\%), Chenopodium album L. (94\%), Echinochloa crus-galli L. (81\%), Thlaspi arvense (82\%) (Fig. 1).

The Zontran CSC effectiveness of action on annual dicotyledonous weeds in dose rates of 0.6 and $0.8 \mathrm{~g} / \mathrm{L}$ (metribuzin 150 and $200 \mathrm{~g} / \mathrm{ha}$ respectively) was comparable with the efficiency of the Analog in the dose rate of $0.5 \mathrm{~kg} / \mathrm{ha}$ (metribuzin $350 \mathrm{~g} / \mathrm{ha}$ ), and at a dose rate of $1.2 \mathrm{~L} / \mathrm{ha}$ (metribuzin $300 \mathrm{~g} / \mathrm{ha}$ ), the effect of Zontran CSC approaches to the Analog effect 


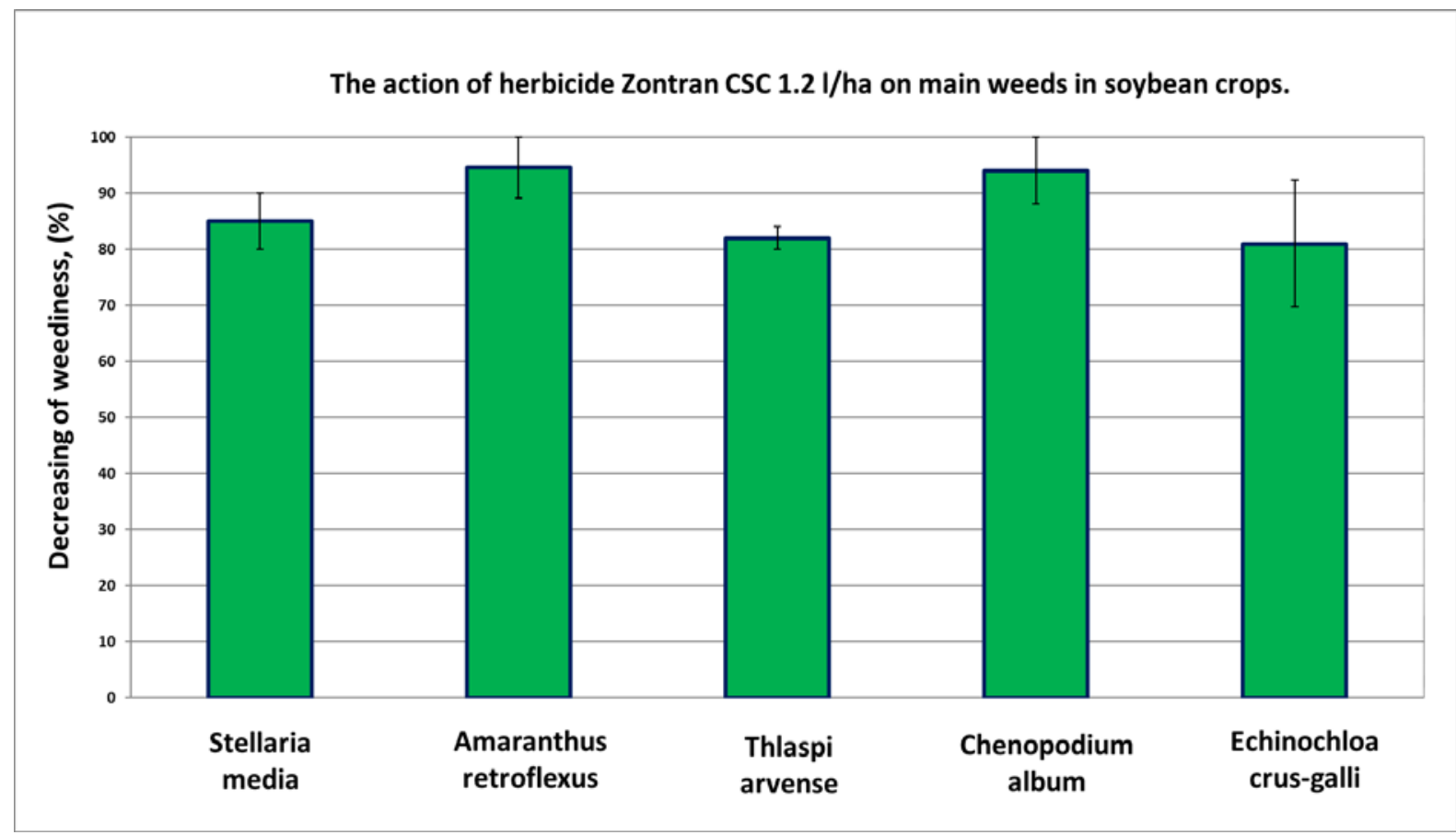

Fig. 1 Efficacy of pre-emergence application of the Zontran CSC herbicide on the main types of weeds in soybean crops.

in a dose rate of $1 \mathrm{~kg} / \mathrm{ha}$ (metribuzin $700 \mathrm{~g} / \mathrm{ha}$ ) (Fig. 2).

By the effectiveness of the action on annual grass weeds, the use of the herbicide Zontran CSC in dose rates of 0.8 and $1.2 \mathrm{~L} / \mathrm{ha}$ was comparable to the efficiency of the Analog in the dose rates of $0.5 \mathrm{~kg} / \mathrm{ha}$ (metribuzin $350 \mathrm{~g} / \mathrm{ha}$ ) and $1 \mathrm{~kg} / \mathrm{ha}$ (metribuzin 700 $\mathrm{g} / \mathrm{ha}$ ), in the dose rate of $0.6 \mathrm{~L} / \mathrm{ha}$ the effectiveness was lower (Fig. 3).

In variants with the Zontran CSC application of 0.8 and $1.2 \mathrm{~L} /$ ha the weight loss of annual dicotyledonous weeds was $98-100 \%$, the weight of annual grasses species-94-100\%, which corresponded to efficiency of Analog in the dose rate of $1 \mathrm{~kg} / \mathrm{ha}$. Weight loss of weeds with the use of Zontran CSC of $0.6 \mathrm{~L} / \mathrm{ha}$ was at the level of Analog efficiency of $0.5 \mathrm{~kg} / \mathrm{ha}$, which was $89-98 \%$ for annual dicotyledonous weeds and $88-93 \%$ for grasses.

Elimination of competition with weeds in the initial period of crop vegetation allowed saving from 26.1 to $58.7 \%$ of the soybeans harvest an medium weediness of crops (annual dicotyledonous weeds $48-49$ ind. $/ \mathrm{m}^{2}$ (194 g/m²), annual grasses 60-63 ind. $/ \mathrm{m}^{2}\left(360 \mathrm{~g} / \mathrm{m}^{2}\right)$ ), and from 28.2 to $32.9 \%$ with high weediness (annual dicotyledonous weeds 242-256 ind. $/ \mathrm{m}^{2}$ (580 g/m ${ }^{2}$ ), annual grasses 151-154 ind./ $/ \mathrm{m}^{2}$ (315 g/m²)) (Table 1).

By the photon correlation spectroscopy method, the particle size of Zontran CSC was determined as 1.327 nm. A small size of particles carrying the active substance leads to suggesting that the "targeting" of metribuzin delivery of Zontran CSC will be higher than the metribuzin from Analog formulation WP with a particle size of $5 \mathrm{mkm}$. The stability of an emulsion depends on many factors, for example, surface tension, viscosity of the dispersion medium, temperature, particle dispersion, etc. But of all the above, the main factor of emulsion stability is the size of the emulsified particles, that is, its dispersion. When the size of the droplets decreases, the effect of gravitational forces decreases, and the forces that keep them in a stably suspended state begin to prevail [5].

It was proved that the surface tension of a droplet containing the active substance in the CSC form will be less $(37 \mathrm{Mn} / \mathrm{m}$ ) than the WP formulation (46 $\mathrm{mN} / \mathrm{m}$ ), which means that the droplet spreading over the surface and holding it on the plant leaf for CSC will be higher than the WP (Fig. 4). Due to the above 


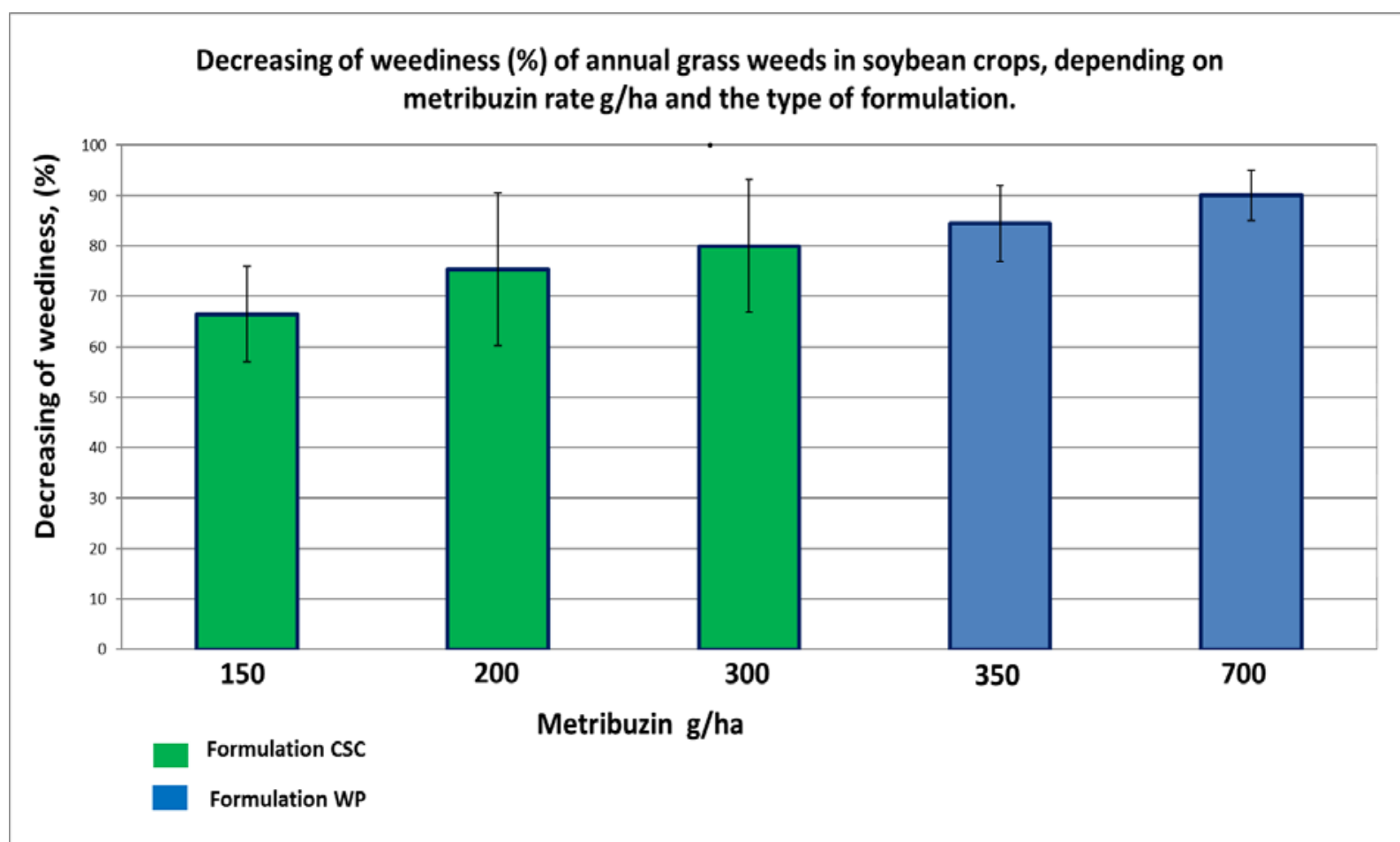

Fig. 2 The biological effectiveness comparison of Zontran CSC and another metribuzin-containing product Analog WP on annual dicotyledonous weeds in soybean crops, depending on the application rate of metribuzin g/ha and the type of formulation.

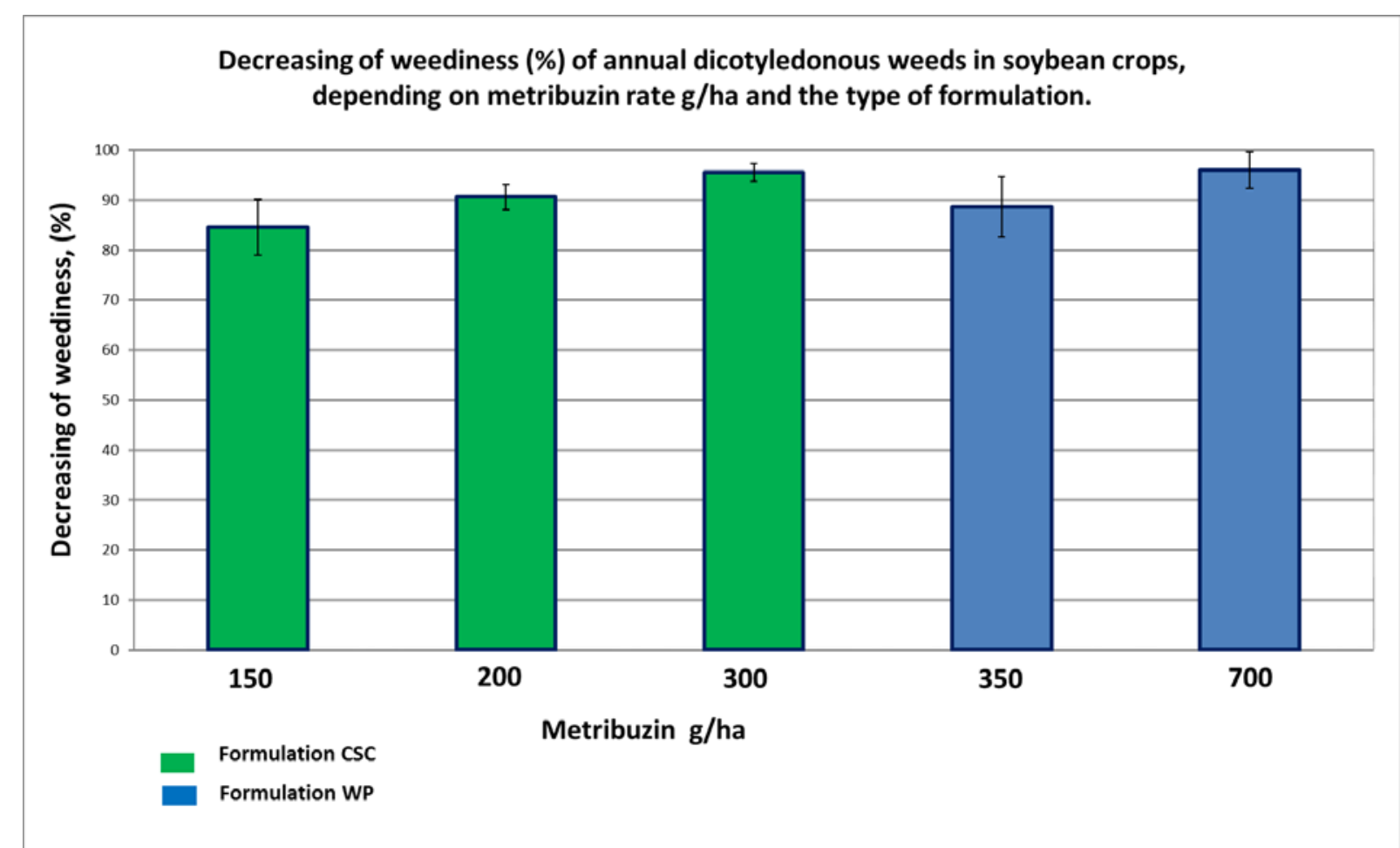

Fig. 3 The biological effectiveness comparison of Zontran CSC and another metribuzin-containing product Analog WP on annual grass weeds in soybean crops, depending on the application rate of metribuzin $\mathrm{g} / \mathrm{ha}$ and the type of formulation. 
Table 1 The influence of herbicides of different formulations on the saved yield of the soybean crops (variety Annushka) with a high and medium degrees of weediness.

\begin{tabular}{llll}
\hline Weediness & \multirow{2}{*}{ Yield in control hundredweight (ha) } & $\begin{array}{l}\text { Saved yield when applied Zontran CSC } \\
1.2 \mathrm{~L} / \mathrm{ha}(\%)\end{array}$ & $\begin{array}{l}\text { Saved yield when applied Analog WP } \\
1 \mathrm{~L} / \mathrm{ha}(\%)\end{array}$ \\
\hline High & 4.6 & $26.1-58.7$ & $32.6-58.7$ \\
Medium & 14.9 & $28.2-32.2$ & $30.2-35.6$ \\
\hline
\end{tabular}

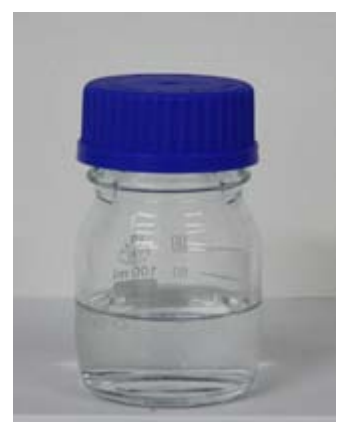

A

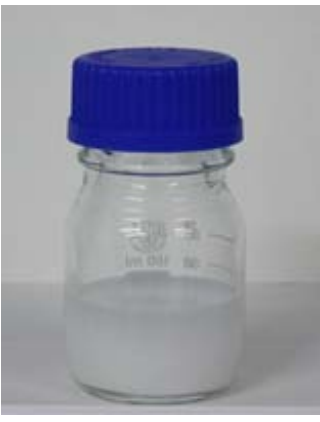

A
Colloidal Solution Concentrate (CSC)

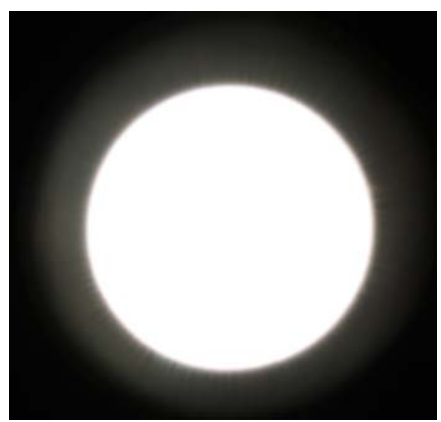

B
Wettable Powder (WP)

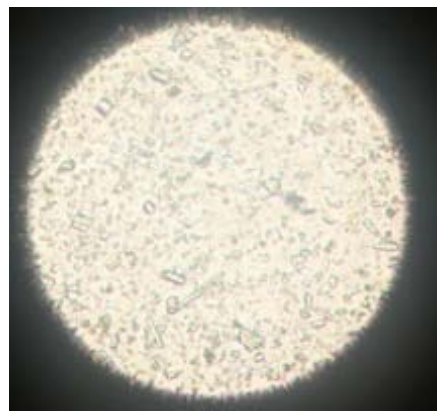

$\mathrm{B}$
C

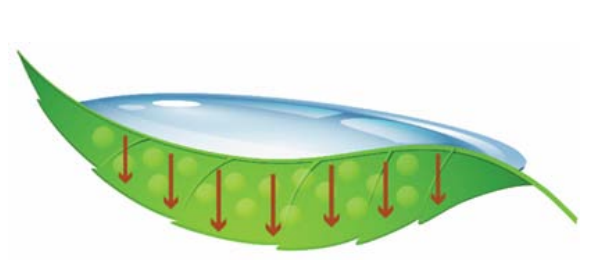

Fig. 4 Comparison of two formulations: CSC and WP.

A: the appearance of the working solution;

$\mathrm{B}$ : a view of the formulation homogeneity by the light microscope $(1,600 \times)$;

C: the intended effect on the surface of the plant leaf.

factors, the efficiency of using the active substance in the CSC formulation will be higher than that in the WP formulation, which allow reducing the dose rate of the active ingredient per unit of cultivated area and, using colloidal formulation, achieve a similar or increased biological efficiency compared to standard forms.

\section{Conclusions}

The effect of application of Zontran CSC on soybean crops at a dose rate of $1.2 \mathrm{~L} / \mathrm{ha}$ was comparable with product contenting at 2.3 times more metribuzin but in WP formulation, while the application rate of metribuzin per 1 ha is $300 \mathrm{~g} / \mathrm{ha}$ and $700 \mathrm{~g} / \mathrm{ha}$, respectively. Thus, the CSC formulation allows reducing the amount of the active substance per unit of cultivated area without loss of biological efficiency and with obtaining reliable yield increase. The product Zontran CSC was included in the State Catalog of Pesticides and Agrochemicals Permitted for Use on the Territory of the Russian Federation [6] in soybean crops with a dose rate of 0.6-1.2 L/ha. 


\section{(Russia) on the Example of the Herbicide Zontran CSC}

\section{Acknowledgement}

The authors express their gratitude to the specialists of JSC Schelkovo Agrohim and All-Russian Institute for Plant Protection (VIZR) who participated in laboratory and field experiments.

\section{References}

[1] Ganiev, M. M., and Nedorezkov, V. D. 2006. Chemical Protection of Plants. Moscow: Kolos.

[2] Roberts, T. R., Hutson, D. H., Lee, P. W., Nicholls, P. H., and Plimmer, J. R. 1998. Metabolic Pathways of
Agrochemicals: Herbicides and Plant Growth Regulators. Royal Society of Chemistry.

[3] Dolzhenko, V. I. 2013. Guidelines for Registration Testing of Herbicides in Agriculture. Ministry of Agriculture RAAS VIZR, St. Petersburg.

[4] Mayevsky, P. F. 2014. Flora of the Middle Zone of the European Part of Russia. 11th ed., Moscow: Partnership KMK Scientific Publications.

[5] Kireev, V. A. 1978. Short Course in Physical Chemistry. 5th ed., Moscow: Stereotypical.

[6] 2018. State Catalog of Pesticides and Agrochemicals, Approved for Use on the Territory of the Russian Federation. Moscow: Agrorus. 